\title{
Un dispositif simple et fiable d'analyse des mouvements de mâchoires chez les petits ruminants
}

\author{
A. DURANTON et L. BUENO * \\ avec la collaboration technique de A. LATOUR \\ Laboratoire de Physiologie, Ecole nationale vétérinaire \\ 23, chemin des Capelles, $F 31076$ Toulouse \\ I.N.R.A., * Station de Pharmacologie-Toxicologie \\ 180, chemin de Tournefeuille, $F 31300$ Toulouse
}

\begin{abstract}
Résumé
Cette note décrit une technique simple et peu onéreuse d'enregistrement des mouvements de mâchoires chez le mouton, adaptable à d'autres espèces. L'originalité réside dans la transformation directe des mouvements de mâchoires en variations de résistance et partant d'un signal électrique. Pour cela un film semi-conducteur en PVC fixé sur une bande de cuir est monté en arche sur une bande élastique faisant partie de la muserole d'un licol.

Un tel système qui ne fait appel qu'à un montage électronique très simple connecté à un enregistreur potentiométrique élimine l'emploi de capteur de pression et a été testé puis utilisé pendant au moins 1500 heures sans problèmes en stabulation. Le signal permet de distinguer les mouvements de préhension et de mastication des aliments et se prête au traitement numérique direct par microprocesseur.
\end{abstract}

\section{Introduction}

L'enregistrement des mouvements de mâchoires a longtemps fait appel à des dispositifs pneumatiques transmettant des variations de pression. Celles-ci sont créées à l'intérieur d'un pneumographe placé sur la muserole d'un licol (DucKworTH \& ShIRlaw, 1955) ou d'un ballonnet placé en région sous-mandibulaire (RUCKEBUSCH, 1963), par déplacement de la mandibule. Ces variations de pression sont transmises à un tambour-inscripteur de Marey porteur d'un stylet inscrivant sur un papier noirci à la fumée se déroulant sur un kymographe.

L'enregistrement graphique obtenu ou gnathogramme permet selon la vitesse de défilement du papier soit l'analyse de la fréquence de mastication sur un temps 
très court soit celui du comportement alimentaire sur $24 \mathrm{~h}$ mais ces deux paramètres comportementaux ne peuvent pas être étudiés simultanément avec un tel dispositif.

Il en est de même des systèmes autonomes développés pour enregistrer le comportement alimentaire des brebis au pâturage dans lesquels les variations de pression sous-mandibulaires assurent le déplacement d'un piston dans une seringue en verre, celui-ci actionnant un stylet inscrivant en spirale sur un disque barosensible les périodes de mouvements masticatoires (RucKebusch \& Bueno, 1973).

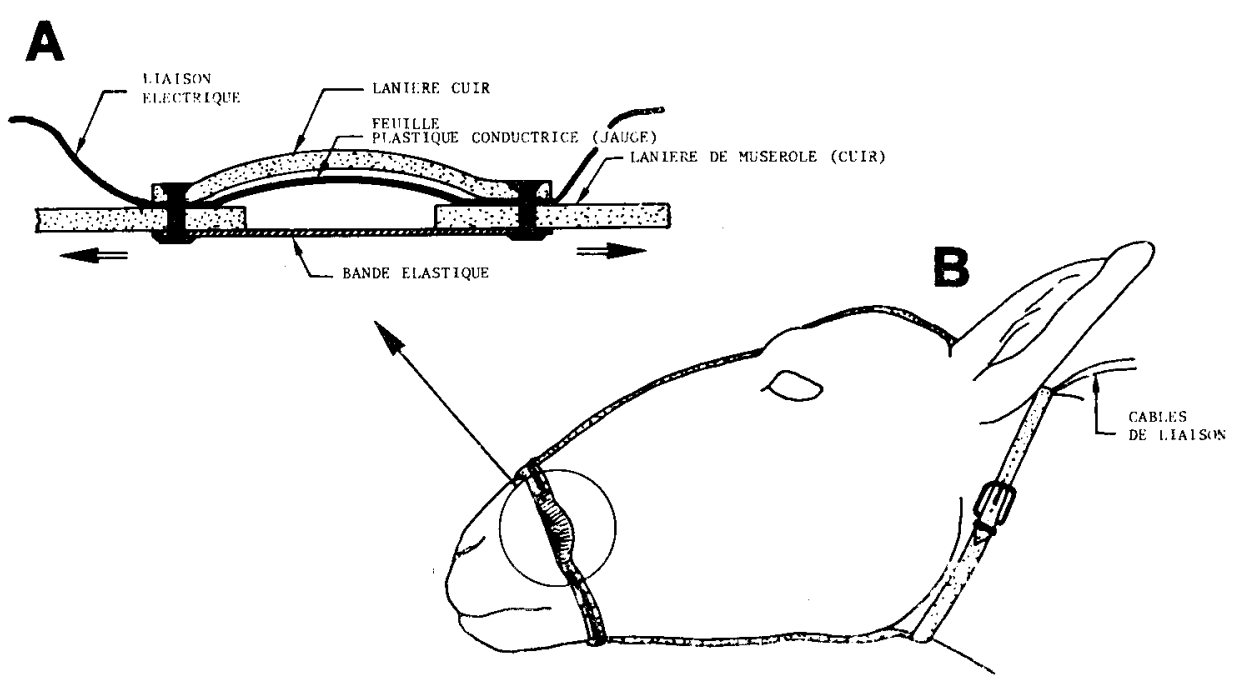

FIG. 1

Schéma d'insertion du capteur dans le licol et situation au cours d'enregistrement.

Scheme of transducer insertion into the recorder and position during recording.

Le capteur constitué par une simple bande de PVC thermoplastique conducteur (ABEY) est alimenté par 2 fils reliés au pont de Wheatstone. Chaque abaissement de la mandibule entraîne un changement de courbe de la bande PVC conductrice.

The transducer constituted of a small piece of semi-conductor PVC film is powered by two cables connected with a Wheatstone's bridge. Each downward movement of the jaw leads to a change in the curve of the semi-conductor PVC film.

Liaison électrique : electrical connection.

Lanière cuir : leather band.

Lanière de muserole (cuir) : nose band (leather).

Bande élastique : elastic band.

Feuille plastique conductrice (jauge) : PVC conductor film (gauge).

Câbles de liaison: connection cables.

La transformation des variations de pression en courant électrique permet une analyse fine des mouvements, leur mémorisation sur bande et leur traitement. Elle peut être réalisée de façon onéreuse en utilisant des chaînes de mesures de pression (Ruckebusch \& Bueno, 1972). Plus récemment il a été proposé de remplacer ce 
matériel par un capteur de déplacement d'un plus faible prix de revient mais qui impose toujours la présence d'un élément transformant les mouvements de la mandibule en variations de pression (LAW \& SuDWEEKs, 1975).

Le dispositif que nous proposons transforme directement les mouvements mandibulaires en variations de courant par l'intermédiaire d'un élément résistif faisant partie du licol.

\section{Description}

Une partie de la muserole d'un licol est rendue extensible par l'introduction d'une bande élastique (fig. $1 \mathrm{~A}$ ). Celle-ci supporte une bande de cuir montée en arche à laquelle est fixée une bande de PVC graphite thermoplastique (Abey) normalement utilisée comme écran de faradisation pour les blindages de conducteurs haute fréquence.

Les contacts électriques sont réalisés à partir des extrémités de fils conducteurs solidaires des rivets de fixation; ces fils sont collés ou cousus sur chaque sangle de liaison de la muserole au collier (fig. $1 \mathrm{~B}$ ).

L'extrémité libre des fils conducteurs est reliée aux bornes d'entrée d'un pont de Wheatstone alimenté soit par une pile $(+4,5 \mathrm{~V})$ soit par une alimentation stabilisée $(+5 \mathrm{~V})$, le montage présenté prévoyant une alimentation par 2 voies d'enregistrements (fig. 2). Les variations de tensions recueillies aux bornes du pont correspondant aux variations de résistance de la partie conductrice de la muserole, sont appliquées aux bornes d'entrée $\mathrm{DC}$ ou $\mathrm{AC}$ d'un enregistrement galvanométrique ou potentiométrique.

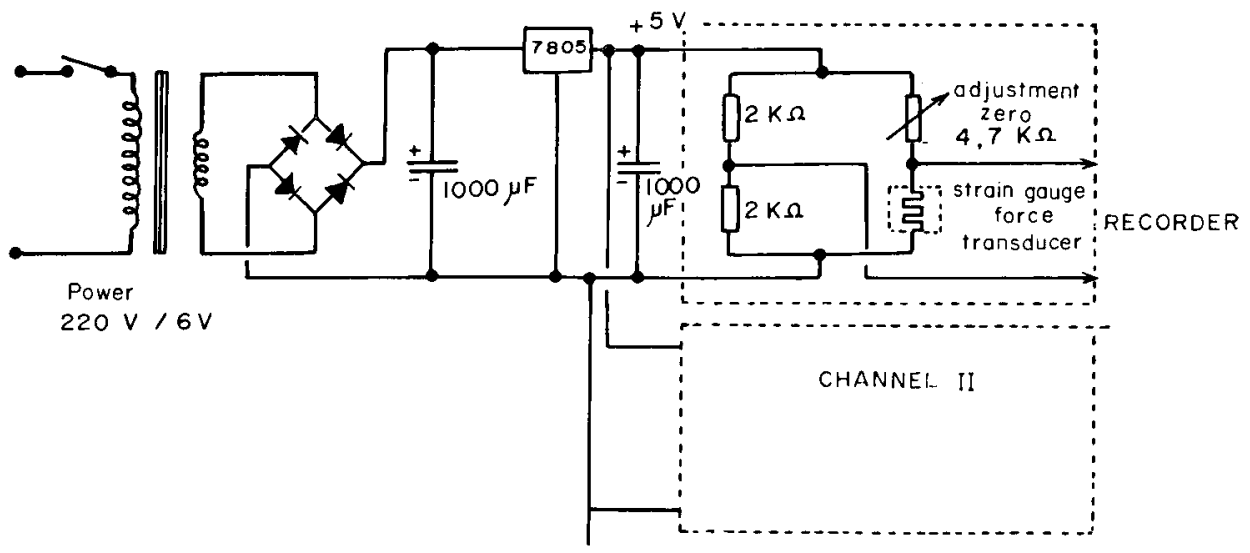

FIG. 2

Circuit de détection et d'amplification des variations de résistance de la jauge.

Circuit of detection and amplification of variations in the resistance of the gauge.

Le signal de sortie est appliqué aux bornes d'un enregistreur potentiométrique ou galvanométrique.

The output signal is directly applied to a potentiometric or galvanometric recorder. 
Un circuit régulateur 7805 assure la stabilisation de la tension appliquée aux bornes du pont éliminant les phénomènes de dérives pour une entrée de type DC.

\section{Utilisation}

Par rapport à un système classique faisant appel aux variations de pression sous-mandibulaire, le dispositif proposé permet la distinction par l'amplitude et la fréquence des mouvements de préhension et de mastication, les 2 types étant facilement identifiables sur un tracé obtenu à grande vitesse de défilement de l'enregistreur (fig. 3). Le traitement numérique du signal par microprocesseur devrait alors permettre de sélectionner les signaux en fonction de ces critères (amplitude, fréquence) et partant d'analyser le rapport des durées : préhension/mastication dont la signification, en particulier dans les phénomènes de satiété à court terme, n'a jamais été envisagée.

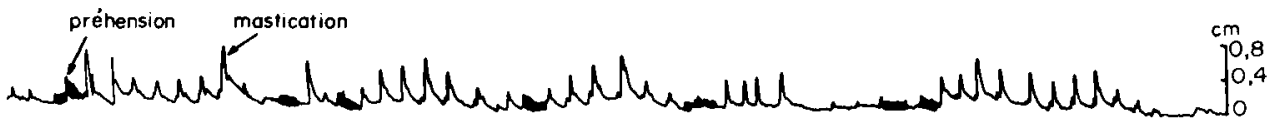

secondes

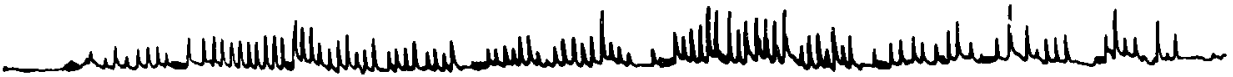

secondes

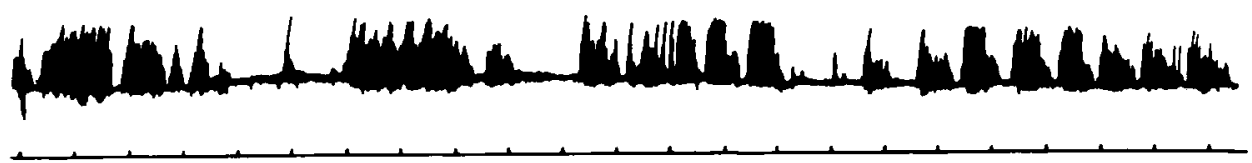

minutes

FIG. 3

Exemples de gnathogrammes obtenus à différentes vitesses de défilement du papier. Gnathogrammes obtained at different tape speeds.

La sensibilité du système permet la distinction entre les mouvements de préhension et de mastication.

Amplitude : élongation de la muserole du licol en $\mathrm{cm}$.

The sensibility of the systems allows to distinguish between gripping and chewing movements amplitude : elongation of the halter in $\mathrm{cm}$.

Préhension : gripping.

Mastication : chewing. 


\section{Discussion} fiabilité.

Un tel dispositif présente les avantages suivants : simplicité, faible coût de revient,

La préparation d'un licol est très rapide, les fils conducteurs sont fixés au licol par des rubans adhésifs, ou mieux sont cousus, les circuits électroniques utilisés sont simples et réalisés à partir d'éléments courants. La présence d'une bande de cuir comme support de la jauge, introduite ainsi dans le licol, est un élément protecteur efficace contre les agressions telles que le grattage et les frottements. La bande plastique conductrice utilisée est par ailleurs très peu sensible aux sécrétions cutanées et salivaires, ainsi qu'à l'immersion lors d'abreuvement.

Compte tenu de la forte résistance de la jauge en Abey (3000-5000 $\Omega$ ) l'alimentation du pont pourra se faire par une pile dont la durée de vie excède 30 jours par voie. Par ailleurs cette grande résistance électrique de la jauge offre la possibilité de monter le pont à une certaine distance de l'animal et partant regrouper dans un même boîtier un nombre relativement élevé de voies. Une telle jauge s'avère également beaucoup moins fragile qu'une jauge classique montée sur un support rigide dont l'élongation limitée empêche l'analyse de l'amplitude des mouvements de mâchoires.

La sortie du type asymétrique est connectée aux bornes d'entrée d'un enregistreur potentiométrique (Philips PM 8010) ou d'un polygraphe du type électroencéphalographe (Alvar, Reega VIII). Dans le cas d'enregistrement à long terme, n'ayant pour objectif que d'évaluer les durées respectives des différentes périodes de prises de nourriture et de rumination au cours du nycthémère, l'élimination des phénomènes de dérive liés à la source d'alimentation, aux variations de valeurs des composants électriques (température ambiante, etc.) ou au placement du licol, pourra être réalisée par l'utilisation d'une amplification du type $\mathrm{AC}$ à longue constante de temps.

Enfin, un tel dispositif permet l'analyse directe par micro-processeur des fréquences et durées de mastication, le signal pouvant être traité directement en temps réel à partir du pont de Wheatstone.

Accepté pour publication en aô̂t 1982.

\section{Summary}

\section{$A$ reliable and simple apparatus to record jaw movements in small ruminants}

A simple device for monitoring jaw movements in small ruminants was developed to record chew rate and duration of rumination and eating. A small piece of semi-conductor PVC film was mounted as an arch in the animal's halter and supported by a rubber band. This semi-conductor plastic film was mounted in a battery powered Wheatstone's bridge. The output signal being directly applied to a potentiometric or galvanometric recorder.

This apparatus has been successfully used as a chew rate monitor in sheep for approximately $1500 \mathrm{~h}$ in feeding behavior studies. Construction advices and a scheme of the apparatus is given in this paper. 


\section{Références bibliographiques}

Duckworth J.E., Shirlaw D.W., 1955. The development of an apparatus to record jaws movements of cattle. Brit. J. Anim. Behav., 3, 5-6.

LAW S.E., Sudweeks E.M., 1975. Electronic transducers for rumination research. J. anim. Sci., 41, 213-218.

RuCKEBusch Y., 1963. Recherches sur le comportement alimentaire des ruminants. Thèse Doct. Sci. Nat. Lyon, $163 \mathrm{p}$.

Ruckebusch Y., Bueno L., 1972. Analyse quantitative des relations activité alimentaireétat de vigilance chez les bovins en stabulation. Ann. Rech. vét., 3, 399-420.

Ruckebusch Y., Bueno L., 1973. Un dispositif simple et autonome d'enregistrement de l'activité alimentaire chez les bovins au pâturage. Ann. Rech. vét., 4, 627-636. 